Research Article

\title{
Gene Expression, Network Analysis, and Drug Discovery of Neurofibromatosis Type 2-Associated Vestibular Schwannomas Based on Bioinformatics Analysis
}

\author{
Qiao Huang $\left(\mathbb{D}\right.$, Si-Jia Zhai $\mathbb{D}^{\text {, }}$, Xing-Wei Liao $(\mathbb{D}$, Yu-Chao Liu, and Shi-Hua Yin $(\mathbb{D}$ \\ Department of Otolaryngology \& Head and Neck Surgery, The Second Affiliated Hospital of Guangxi Medical University, \\ Nanning 530007, China \\ Correspondence should be addressed to Shi-Hua Yin; shihuayin@126.com
}

Received 26 March 2020; Revised 27 May 2020; Accepted 1 June 2020; Published 15 July 2020

Academic Editor: Pierfrancesco Franco

Copyright (c) 2020 Qiao Huang et al. This is an open access article distributed under the Creative Commons Attribution License, which permits unrestricted use, distribution, and reproduction in any medium, provided the original work is properly cited.

Neurofibromatosis Type 2- (NF2-) associated vestibular schwannomas (VSs) are histologically benign tumors. This study aimed to determine disease-related genes, pathways, and potential therapeutic drugs associated with NF2-VSs using the bioinformatics method. Microarray data of GSE108524 were downloaded from the Gene Expression Omnibus (GEO) database, and differentially expressed genes (DEGs) were screened using GEO2R. The functional enrichment and pathway enrichment of DEGs were performed using Gene Ontology (GO) and Kyoto Encyclopedia of Genes Genomes (KEGG). Furthermore, the STRING and Cytoscape were used to analyze the protein-protein interaction (PPI) network of all differentially expressed genes and identify hub genes. Finally, the enriched gene sets belonging to the identified pathways were queried against the Drug-Gene Interaction database to find drug candidates for topical use in NF2-associated VSs. A total of 542 DEGs were identified, including 13 upregulated and 329 downregulated genes, which were mainly enriched in terms of focal adhesion, PI3K-Akt signaling pathway, ECM-receptor interaction, Toll-like receptor signaling pathway, Rap1 signaling pathway, and regulation of actin cytoskeleton. 28 hub genes were identified based on the subset of PPI network, and 31 drugs were selected based on the Drug-Gene Interaction database. Drug discovery using bioinformatics methods facilitates the identification of existing or potential therapeutic drugs to improve NF2 treatment.

\section{Introduction}

VSs, also known as acoustic neuromas, are histologically benign tumors originating from the eighth nerve. NF2 is a rare autosomal dominant inherited disorder tumor caused by deletion or loss-of-function mutations in the NF2 gene encoding merlin [1]. The main characteristic of NF2-associated VSs is the bilateral schwannomas of the vestibular nerve, which leads to sensorineural hearing loss, facial paralysis, vestibular dysfunction, brainstem compression, and even death [2]. Despite their benign nature, NF2-associated VSs have poor prognosis prone to recurrence, and there are no curative treatments. At present, the primary treatments are follow-up observation, microsurgery, and radiosurgery which are not always effective and sometimes cause neurological deficits [3]. Patients with hearing loss sometimes accept the otolaryngology surgery and require improving or saving hearing. With the targeted molecular therapies becoming increasingly common, drug therapy has gradually become possible. Therefore, it is urgently required to determine effective drug targets for NF2-associated VSs therapies. The present study aimed to determine diseaserelated genes, pathways, and potential targeted therapeutic drugs associated with NF2-associated VSs using the bioinformatics method.

\section{Materials and Methods}

2.1. Microarray Datasets. The gene expression profile GSE108524 of the NF2-associated VSs and normal nerve groups was obtained from the NCBI GEO database. These microarray data were based on GPL17586 Platform [HTA- 

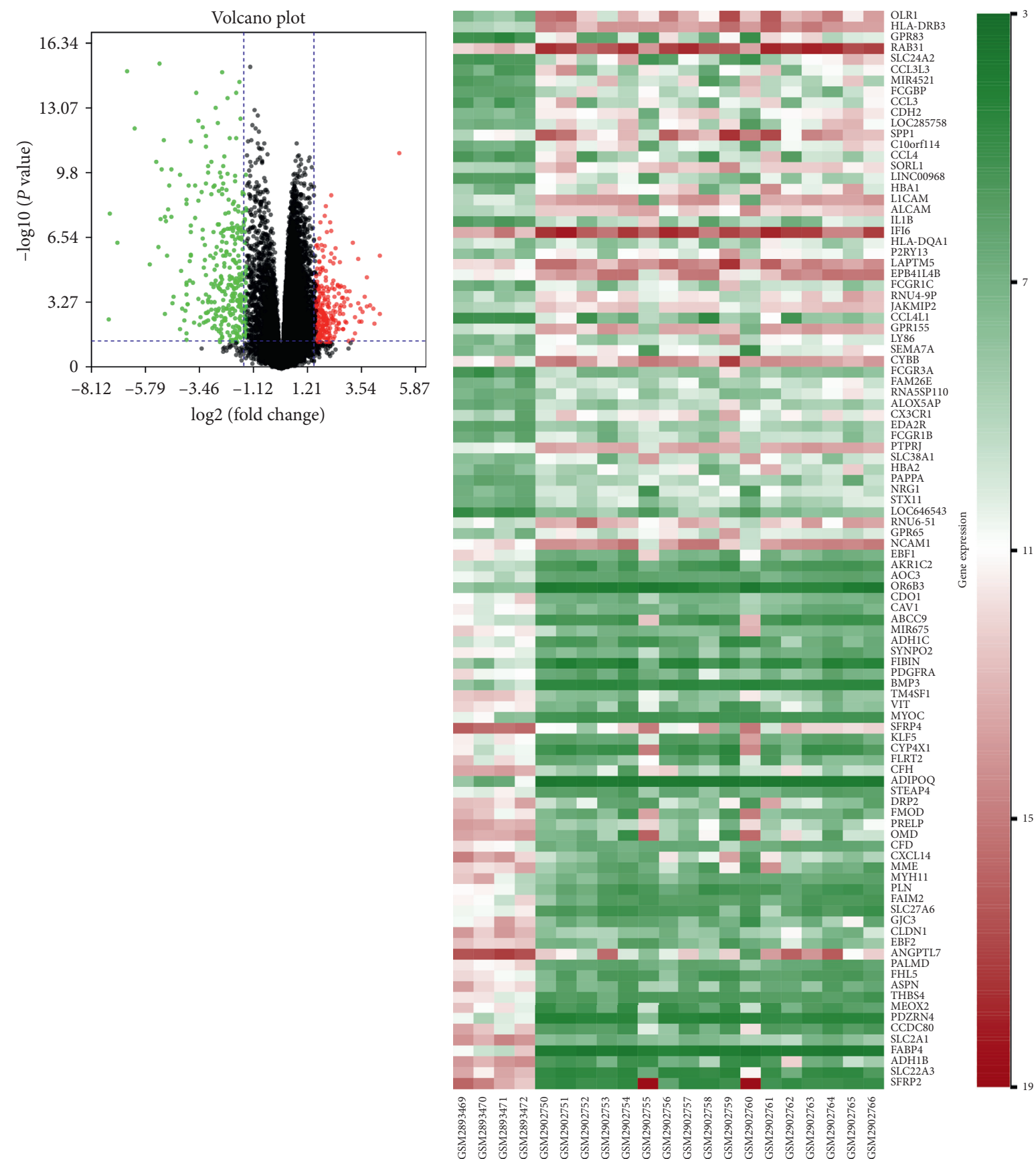

(a)

(b)

FIGURE 1: (a) DEGs were selected by volcano plot filtering ( $\mid$ fold change $\mid \geq 1.5$ and adj. $P<0.05$ ). (b) The heat map of DEGs in NF2-associated VSs (top 100 upregulated and downregulated genes). Green represents a downregulated expression, and red indicates an upregulated level.

2_0] Affymetrix Human Transcriptome Array 2.0 [transcript (gene) version], including 17 NF2-associated VSs tissues and 4 normal nerves.

2.2. Identification of DEGs. GEO2R (http://www.ncbi.nlm. nih.gov/geo/geo2r/), a web tool based on the analysis of variance or $t$-test, was used to identify DEGs between NF2associated VSs tissues and normal nerves. The DEGs were identified as the genes with $|\log \mathrm{FC}| \geq 1.5$ and adj. $P<0.05$.
2.3. Functional and Pathway Enrichment Analysis of DEGs. The Database for Annotation, Visualization, and Integrated Discovery (DAVID) (Version 6.8, https://david.ncifcrf.gov/) was used to perform GO and KEGG pathway enrichment analysis of DEGs. GO analysis contains biological process (BP), cellular component (CC), and molecular function (MF). GO term with the criterion of $P<0.05$ and false discovery rate $(\mathrm{FDR})<0.05$ and $\mathrm{KEGG}$ pathway analysis with the criterion of $P<0.05$ were considered statistically significant. 
TABLE 1: GO analysis of differentially expressed genes.

\begin{tabular}{|c|c|c|c|c|}
\hline Category & Term & Count & $P$ value & FDR \\
\hline $\mathrm{BP}$ & GO:0007155 cell adhesion & 38 & $<0.001$ & $<0.001$ \\
\hline $\mathrm{BP}$ & GO:0006954 inflammatory response & 34 & $<0.001$ & $<0.001$ \\
\hline $\mathrm{BP}$ & GO:0006955 immune response & 32 & $<0.001$ & $<0.001$ \\
\hline $\mathrm{BP}$ & GO:0007165 signal transduction & 58 & $<0.001$ & 0.0054 \\
\hline $\mathrm{BP}$ & GO:0051897 positive regulation of protein kinase B signaling & 12 & $<0.001$ & 0.0203 \\
\hline $\mathrm{BP}$ & GO:0070374 positive regulation of ERK1 and ERK2 cascade & 17 & $<0.001$ & 0.0261 \\
\hline BP & GO:0043547 positive regulation of GTPase activity & 34 & $<0.001$ & 0.0265 \\
\hline $\mathrm{BP}$ & GO:0030198 extracellular matrix organization & 18 & $<0.001$ & 0.0285 \\
\hline $\mathrm{BP}$ & GO:0030335 positive regulation of cell migration & 17 & $<0.001$ & 0.0488 \\
\hline $\mathrm{CC}$ & GO:0005615 extracellular space & 87 & $<0.001$ & $<0.001$ \\
\hline $\mathrm{CC}$ & GO:0005887 integral component of plasma membrane & 88 & $<0.001$ & $<0.001$ \\
\hline $\mathrm{CC}$ & GO:0005886 plasma membrane & 177 & $<0.001$ & $<0.001$ \\
\hline $\mathrm{CC}$ & GO:0005578 proteinaceous extracellular matrix & 31 & $<0.001$ & $<0.001$ \\
\hline $\mathrm{CC}$ & GO:0009986 cell surface & 42 & $<0.001$ & $<0.001$ \\
\hline $\mathrm{CC}$ & GO:0070062 extracellular exosome & 121 & $<0.001$ & $<0.001$ \\
\hline $\mathrm{CC}$ & GO:0005576 extracellular region & 81 & $<0.001$ & $<0.001$ \\
\hline $\mathrm{CC}$ & GO:0031012 extracellular matrix & 28 & $<0.001$ & $<0.001$ \\
\hline $\mathrm{CC}$ & GO:0009897 external side of plasma membrane & 21 & $<0.001$ & $<0.001$ \\
\hline $\mathrm{CC}$ & GO:0045121 membrane raft & 20 & $<0.001$ & 0.0020 \\
\hline CC & GO:0016021 integral component of membrane & 179 & $<0.001$ & 0.0020 \\
\hline $\mathrm{CC}$ & GO:0005925 focal adhesion & 27 & $<0.001$ & 0.0122 \\
\hline CC & GO:0045202 synapse & 17 & $<0.001$ & 0.0224 \\
\hline MF & GO:0008201 heparin binding & 20 & $<0.001$ & $<0.001$ \\
\hline MF & GO:0005178 integrin binding & 14 & $<0.001$ & 0.0041 \\
\hline
\end{tabular}

2.4. Protein-Protein Interaction (PPI) Network Analysis. We submitted DEGs in Search Tool for the Retrieval of Interacting Genes database (STRING, http://www.string-db. org/) to screen the PPI pairs with a combined score of $\geq 0.4$ and visualized the interaction using Cytoscape software (Version 3.7.0.). Finally, CentiScaPe and Molecular Complex Detection (MCODE), a Cytoscape plugin, were utilized to screen PPI network key genes. The default parameters of MCODE were used: degree cutoff $\geq 2$, node score cutoff $\geq 0.2$, $\mathrm{k}$-score $\geq 2$, and maximum depth $=100$.

2.5. Drug-Gene Interaction Analysis. To better identify potential targeted therapeutic drugs for NF2-associated VSs, the hub genes were mapped onto the Drug-Gene Interaction database (DGIdb; http://www.dgidb.org) to obtain potential drug target genes and potential NF2-associated VSs treatment drugs. Visualization of the drug-gene interaction was generated using Cytoscape software (Version 3.7.0.). In addition, ClinicalTrials.gov (https://clinicaltrials.gov) was used to identify whether drugs have been previously investigated or are being currently tested in clinical trials.

2.6. Human NF2-Associated VSs Specimens. Human NF2associated VSs tissues with the matched normal adjacent specimens were obtained from the Second Hospital of Guangxi Medical University. This study was approved by the Ethics Committee of the Second Hospital of Guangxi Medical University.

2.7. Quantitative PCR ( $q P C R$ ). Reverse transcription was carried out using SYBR premix EX Taq (Takara, Japan), and
SYBR Premix Ex Taq II (Takara) was used for qPCR. We used several sequences: EGFR forward primer $5^{\prime}$-CTACAACCCCACCACGTACC- $3^{\prime}$ and reverse primer $5^{\prime}$ CGCACTTCTTACACTTGCGG-3'; GAPDH forward primer $5^{\prime}$-CTTCGCTCTCTGCTCCTCCTGTTCG-3' $3^{\prime}$ and reverse primer $5^{\prime}$-ACCAGGCGCCCAATACGACCAAAT3 . The results were calculated using the $2^{-\Delta \Delta \mathrm{Ct}}$ method.

2.8. Statistical Analysis. Statistical analysis was conducted by SPSS 20.0 software. The statistical significance between groups was determined using a two-tailed Student's $t$-test. Values of $P<0.05$ were considered to indicate statistically significant differences.

\section{Results}

3.1. Identification of DEGs. A total of 542 DEGs, including 13 upregulated and 329 downregulated genes, were identified by comparing $17 \mathrm{NF} 2$-associated VSs tissues and 4 normal nerves from GSE108524. The heat map and volcano plot showed these DEGs (Figure 1).

\subsection{Functional Annotation and Pathway Enrichment Analysis} of DEGs. GO functional annotation revealed that the DEGs were significantly enriched in BP terms including cell adhesion, inflammatory response, immune response, signal transduction, positive regulation of protein kinase $B$ signaling, positive regulation of ERK1 and ERK2 cascade, and positive regulation of GTPase activity. In addition, the CC terms mainly showed plasma membrane, extracellular exosome, extracellular region, extracellular matrix, and membrane raft. MF enrichment indicated heparin binding 
TABLE 2: KEGG pathway analysis of differentially expressed genes.

\begin{tabular}{|c|c|c|c|}
\hline Term & Count & $\begin{array}{c}P \\
\text { value }\end{array}$ & Genes \\
\hline $\begin{array}{l}\text { hsa05150: staphylococcus aureus } \\
\text { infection }\end{array}$ & 13 & $<0.001$ & $\begin{array}{c}\text { C3AR1, C3, HLA-DRB3, FPR1, C1R, C1S, HLA-DQA1, FCGR1A, CFH, } \\
\text { FCGR3A, CFD, SELPLG, FCGR3B }\end{array}$ \\
\hline hsa04145: phagosome & 19 & $<0.001$ & $\begin{array}{c}\text { MRC1, NOS1, OLR1, C3, TUBB2A, HLA-DRB3, TLR2, HLA-C, C1R, TLR6, } \\
\text { HLA-DQA1, CYBB, CD36, FCGR1A, COMP, CLEC7A, FCGR3A, FCGR3B, } \\
\text { THBS4 }\end{array}$ \\
\hline $\begin{array}{l}\text { hsa04514: cell adhesion molecules } \\
\text { (CAMs) }\end{array}$ & 16 & $<0.001$ & $\begin{array}{l}\text { CLDN19, HLA-DRB3, HLA-C, L1CAM, NLGN3, CDH2, HLA-DQA1, CDH5, } \\
\text { ALCAM, NCAM1, CD86, CD34, ITGA8, CLDN1, CD4, SELPLG }\end{array}$ \\
\hline hsa04640: hematopoietic cell lineage & 12 & $<0.001$ & $\begin{array}{l}\text { CR1, CD37, CD36, CD34, HLA-DRB3, FCGR1A, MME, IL1B, CD4, ANPEP, } \\
\text { CSF2RA, CSF1R }\end{array}$ \\
\hline hsa05144: malaria & 9 & $<0.001$ & CR1, CD36, COMP, TLR2, IL1B, HBA2, HBA1, HBB, THBS4 \\
\hline $\begin{array}{l}\text { hsa04610: complement and } \\
\text { coagulation cascades }\end{array}$ & 10 & $<0.001$ & C3AR1, VWF, CR1, C3, F13A1, CFH, TFPI, C1R, C1S, CFD \\
\hline hsa04510: focal adhesion & 17 & 0.0017 & $\begin{array}{l}\text { PIK3CG, EGFR, CAV1, TNXB, TNC, FLNB, MYL9, VWF, CCND1, PAK3, } \\
\text { CCND2, COMP, ITGA8, COL6A3, PDGFRA, SPP1, THBS4 }\end{array}$ \\
\hline $\begin{array}{l}\text { hsa04060: cytokine-cytokine receptor } \\
\text { interaction }\end{array}$ & 18 & 0.0022 & $\begin{array}{l}\text { EGFR, CCL3, TGFBR1, LIFR, EDA2R, CCL4L1, CCL4, CXCL12, IL17RA, LEP, } \\
\text { PPBP, CXCL14, CCL3L3, CX3CR1, PDGFRA, IL1B, CSF2RA, CSF1R }\end{array}$ \\
\hline hsa05140: leishmaniasis & 9 & 0.0027 & CR1, C3, HLA-DRB3, FCGR1A, TLR2, IL1B, FCGR3A, FCGR3B, HLA-DQA1 \\
\hline hsa04151: PI3K-akt s & 23 & 0.0033 & $\begin{array}{r}\text { EGFR, PIK3CG, FGF7, TNXB, TNC, TLF } \\
\text { LPAR5, CCND2, COMP, ITGA8, COL6 } \\
\text { SPP1, THB }\end{array}$ \\
\hline hsa00350: tyrosine metabolism & 6 & 0.0063 & MAOA, AOX1, ADH1C, AD \\
\hline lway & 8 & & LPL, CD36, OLR1, PLIN1, SLC27A6, FABP4, \\
\hline & 9 & & VWF, CD36, TNXB, COMP, TNC, ITGA8, COL6A3, SPP1, THBS4 \\
\hline $\begin{array}{l}\text { hsa04620: Toll-like receptor signaling } \\
\text { pathway }\end{array}$ & 10 & 0.0100 & PIK3CG, CD86, CCL3, CCL3L3, TLR2, CCL4L1, IL1B, TLR6, CCL4, SPP1 \\
\hline hsa05323: rheumatoid arthritis & 9 & 0.0101 & $\begin{array}{l}\text { CD86, CCL3, HLA-DRB3, CCL3L3, TLR2, IL1B, ANGPT1, CXCL12, HLA- } \\
\text { DQA1 }\end{array}$ \\
\hline hsa05218: melanoma & 8 & 0.0103 & PIK3CG, EGFR, CCND1, FGF7, PDGFRA, MD \\
\hline hsa04015: Rap1 signaling pathway & 15 & 0.0126 & $\begin{array}{l}\text { FYB, PIK3CG, EGFR, FGF7, FPR1, FGF10, APBB1IP, DOCK4, PLCB4, LPAR5, } \\
\text { RASGRP3, PDGFRA, ANGPT1, FGF1, CSF1R }\end{array}$ \\
\hline & 7 & & CAV1, CD86, CCND1, HLA-DRB3, SGCD, HLA-C, HLA-DQA1 \\
\hline & r & & $\begin{array}{c}\text { PLA2G4A, PLCB4, NOS1, GRIA2, LYN, GUCY1A2, GUCY1B3 } \\
\text { EGFR, TNXB, CYP1B1, TNC, MIRLET7F1, MIR99A, ZEB1, MIR222, MIR221, }\end{array}$ \\
\hline hsa05206: microRNAs in cancer & 18 & 0.0176 & $\begin{array}{l}\text { IRS1, DDIT4, NOTCH3, CCND1, CCND2, PDGFRA, MDM2, MARCKS, } \\
\text { MIR181B2 }\end{array}$ \\
\hline hsa05152: tuberculosis & 13 & 0.0178 & $\begin{array}{l}\text { MRC1, CR1, ITGAX, C3, FCGR1A, HLA-DRB3, TLR2, IL1B, CLEC7A, FCGR3A, } \\
\text { TLR6, FCGR3B, HLA-DQA1 }\end{array}$ \\
\hline hsa05205: proteoglycans in cancer & 14 & 0.0191 & $\begin{array}{c}\text { PIK3CG, EGFR, CAV1, LUM, FZD1, TLR2, DCN, FLNB, CCND1, CBLB, GPC3, } \\
\text { RRAS2, MDM2, PTCH1 }\end{array}$ \\
\hline & & & PLCB4, IL \\
\hline & 5 & & CD86, HLA-DRB3, IL1B, HLA-C, HLA-DQA1 \\
\hline $\begin{array}{l}\text { hsa05142: Chagas disease (American } \\
\text { trypanosomiasis) }\end{array}$ & 9 & 0.0254 & PIK3CG, CCL3, PLCB4, C3, TGFBR1, CCL3L3, TLR2, IL1B, TLR6 \\
\hline hsa05200: pathways in cancer & 22 & 0.0267 & $\begin{array}{c}\text { EGFR, PIK3CG, FGF7, TGFBR1, FZD1, RUNX1T1, FGF10, CXCL12, CBLB, } \\
\text { CCND1, PLCB4, LPAR5, RASGRP3, SLC2A1, PDGFRA, MDM2, PTCH1, } \\
\text { PTCH2, HHIP, FGF1, CSF2RA, CSF1R }\end{array}$ \\
\hline $\begin{array}{l}\text { hsa04810: regulation of actin } \\
\text { cytoskeleton }\end{array}$ & 14 & 0.0282 & $\begin{array}{c}\text { PIK3CG, EGFR, FGF7, FGF10, NCKAP1L, MYL9, ARPC1B, ITGAX, CHRM3, } \\
\text { PAK3, ITGA8, RRAS2, PDGFRA, FGF1 }\end{array}$ \\
\hline hsa04380: osteoclast differentiation & 10 & 0.0349 & $\begin{array}{l}\text { PIK3CG, CYBB, FCGR1A, TGFBR1, IL1B, FCGR3A, TREM2, FCGR3B, CSF1R, } \\
\text { BLNK }\end{array}$ \\
\hline
\end{tabular}

and integrin binding (Table 1). Furthermore, KEGG pathway enrichment analysis revealed focal adhesion, PI3K-Akt signaling pathway, ECM-receptor interaction, Toll-like receptor signaling pathway, Rap1 signaling pathway, and regulation of actin cytoskeleton (Table 2).

3.3. PPI Network Analysis. In total, we made the PPI network of 369 nodes and 1,322 edges, based on the
STRING database (Figure 2(a)). We identified 28 hub genes with connectivity degree $\geq 20$ (Figure 2(b), Table 3 ). Then, using MCODE, three modules with scores $>4.5$ and a number of nodes $>18$ were selected. Module 1 with a score of 9.368 consisted of 20 nodes and 89 edges (Figure 2(c)), module 2 with a score of 4.588 comprised 18 nodes and 39 edges (Figure 2(d)), and module 3 with a score of 4.455 comprised 23 nodes and 49 edges (Figure 2(e)). 


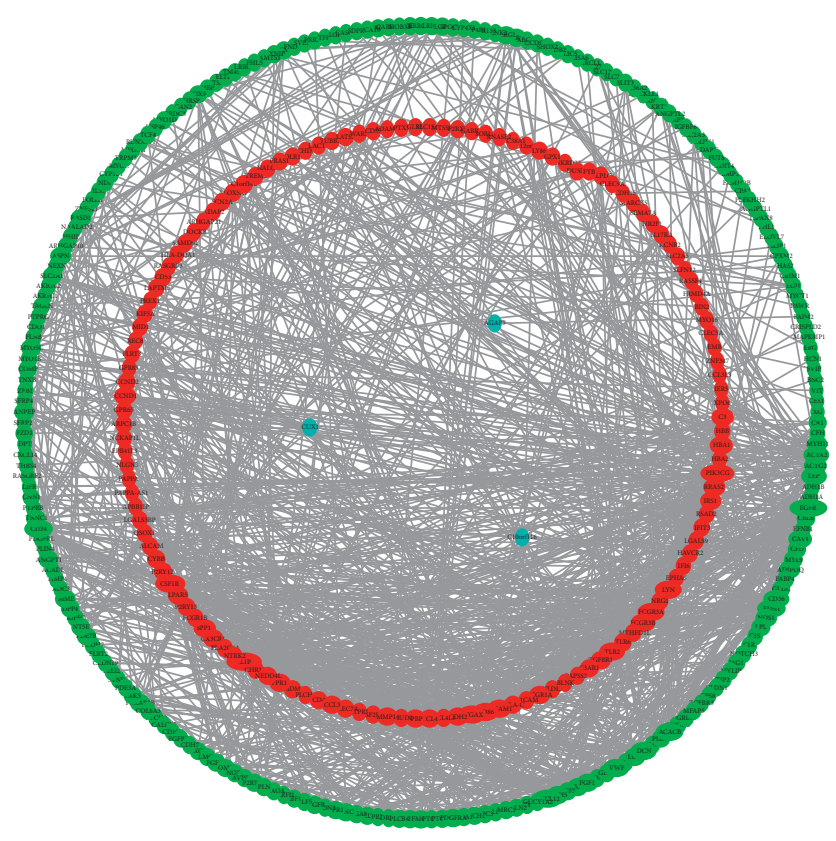

(a)

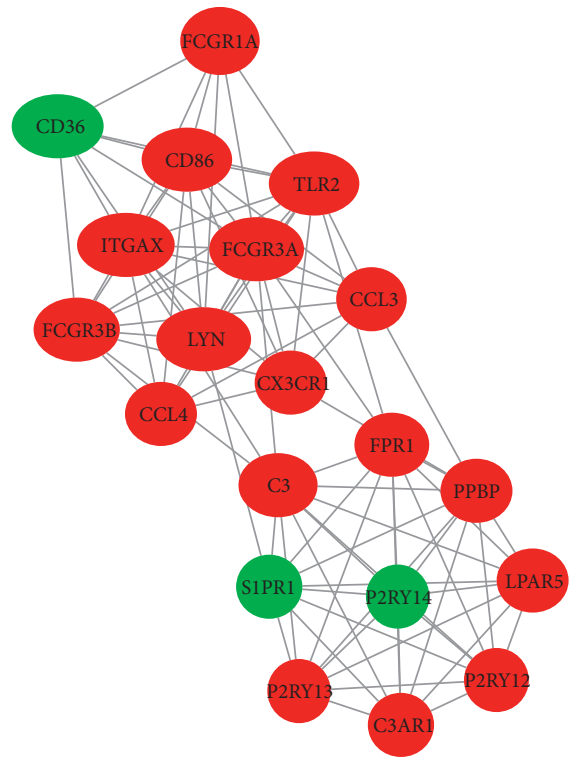

(c)

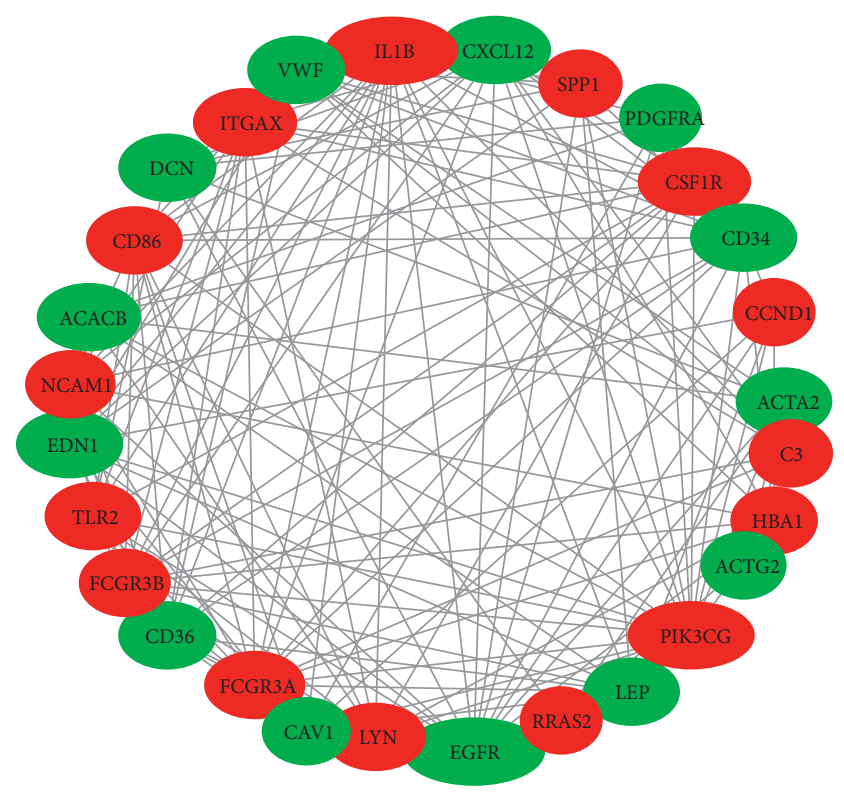

(b)

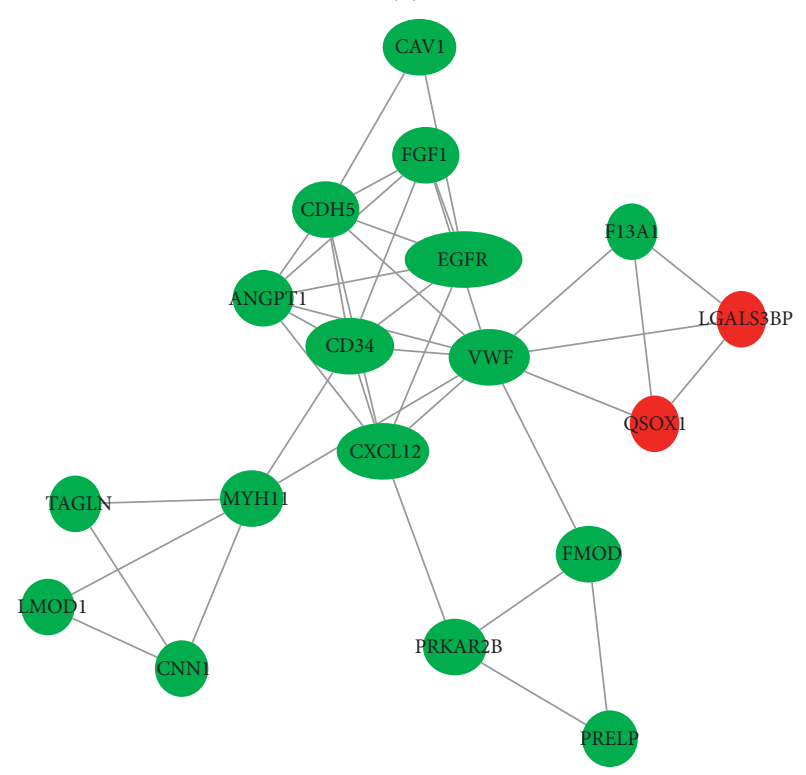

(d)

Figure 2: Continued. 


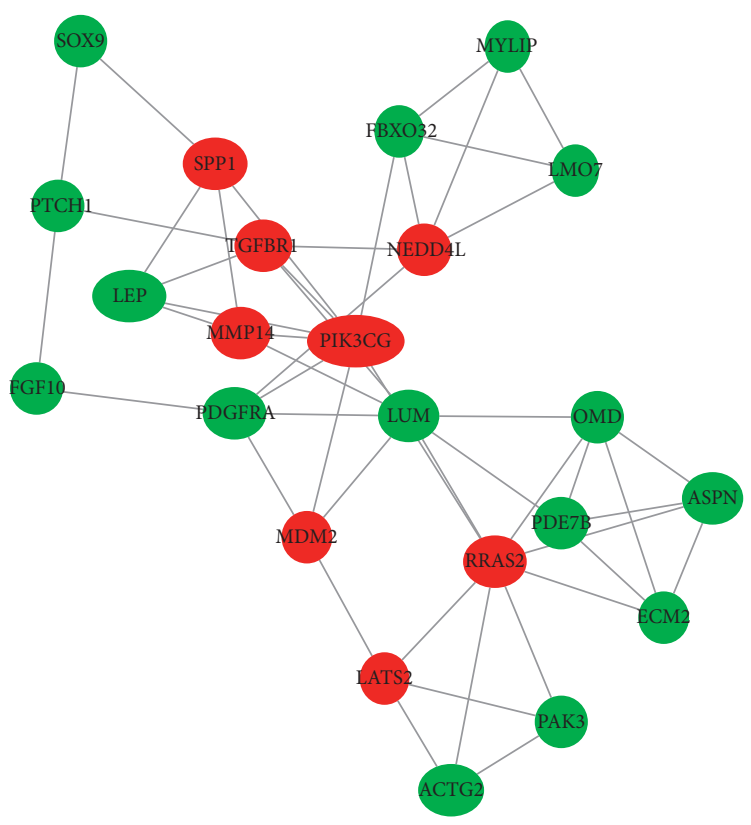

(e)

Figure 2: (a) The PPI network of DEGs. (b) The hub genes with connectivity degree $\geq 20$. (c) Module 1. (d) Module 2. (e) Module 3. Green represents a downregulated expression, and red indicates an upregulated level.

3.4. Drug-Gene Interaction Analysis. Based on the DGIdb, we use the 28 hub genes to screen for drug-gene interactions, which revealed that 31 drugs associated with 12 key genes may be potential NF2 treatment drugs (Figure 3). Based on ClinicalTrials.gov, we found that nilotinib was previously investigated for Phase 2 of growing VSs treatment and everolimus is being used in Phase 2 of the NF2 treatment study.

3.5. mRNA Expression Levels of EGFR. qPCR analysis verified EGFR mRNA underexpression levels in the NF2-associated VSs tissues (Figure 4).

\section{Discussion}

In this study, we found that the 28 hub genes had been insufficiently studied or not studied at all in VSs, 12 of which may be target genes for potential NF2 treatment drugs. Among these genes, IL1B, PIK3CG, CSF1R, LYN, FCGR3A, FCGR3B, SPP1, and CCND1 were upregulated in NF2-associated VSs, while EGFR, DCN, VWF, and PDGFRA were downregulated. Then, LYN, FCGR3A, and FCGR3B are involved in "module 1 " of the subnetwork, in which GO functional annotation is enriched in inflammatory response and immune response, and KEGG pathway enrichment analysis is enriched in staphylococcus aureus infection, phagosome, and osteoclast differentiation. EGFR and VWF are involved in "module 2," which is enriched in focal adhesion and PI3K-Akt signaling pathway. PIK3CG and SPP1 are involved in "module 3," which is also enriched in focal adhesion and PI3K-Akt signaling pathway.

We found that upregulated genes PIK3CG, CSF1R, SPP1, and CCND1 and downregulated genes EGFR and
TABle 3: 28 hub genes with connectivity degree $\geq 20$.

\begin{tabular}{|c|c|c|c|}
\hline Number & Gene & Degree of connectivity & Regulation \\
\hline 1 & EGFR & 59 & Down \\
\hline 2 & IL1B & 53 & $\mathrm{Up}$ \\
\hline 3 & PIK3CG & 49 & Up \\
\hline 4 & CSF1R & 40 & Up \\
\hline 5 & CXCL12 & 39 & Down \\
\hline 6 & CD34 & 36 & Down \\
\hline 7 & EDN1 & 36 & Down \\
\hline 8 & ITGAX & 34 & Up \\
\hline 9 & ACACB & 34 & Down \\
\hline 10 & LYN & 32 & $\mathrm{Up}$ \\
\hline 11 & FCGR3A & 32 & $\mathrm{Up}$ \\
\hline 12 & DCN & 30 & Down \\
\hline 13 & CD36 & 30 & Down \\
\hline 14 & VWF & 30 & Down \\
\hline 15 & CD86 & 29 & Up \\
\hline 16 & TLR2 & 29 & $\mathrm{Up}$ \\
\hline 17 & ACTA2 & 29 & Down \\
\hline 18 & LEP & 29 & Down \\
\hline 19 & FCGR3B & 26 & $\mathrm{Up}$ \\
\hline 20 & NCAM1 & 25 & Up \\
\hline 21 & CAV1 & 24 & Down \\
\hline 22 & HBA1 & 23 & Up \\
\hline 23 & ACTG2 & 22 & Down \\
\hline 24 & SPP1 & 21 & Up \\
\hline 25 & C3 & 21 & $\mathrm{Up}$ \\
\hline 26 & PDGFRA & 20 & Down \\
\hline 27 & CCND1 & 20 & $\mathrm{Up}$ \\
\hline 28 & RRAS2 & 20 & Up \\
\hline
\end{tabular}

VWF were significantly enriched in PI3K-Akt signaling pathway involved in VSs development, which can increase schwannoma cell proliferation, survival, and cell-matrix adhesion acting [4-6]. That may be the cause of poor 


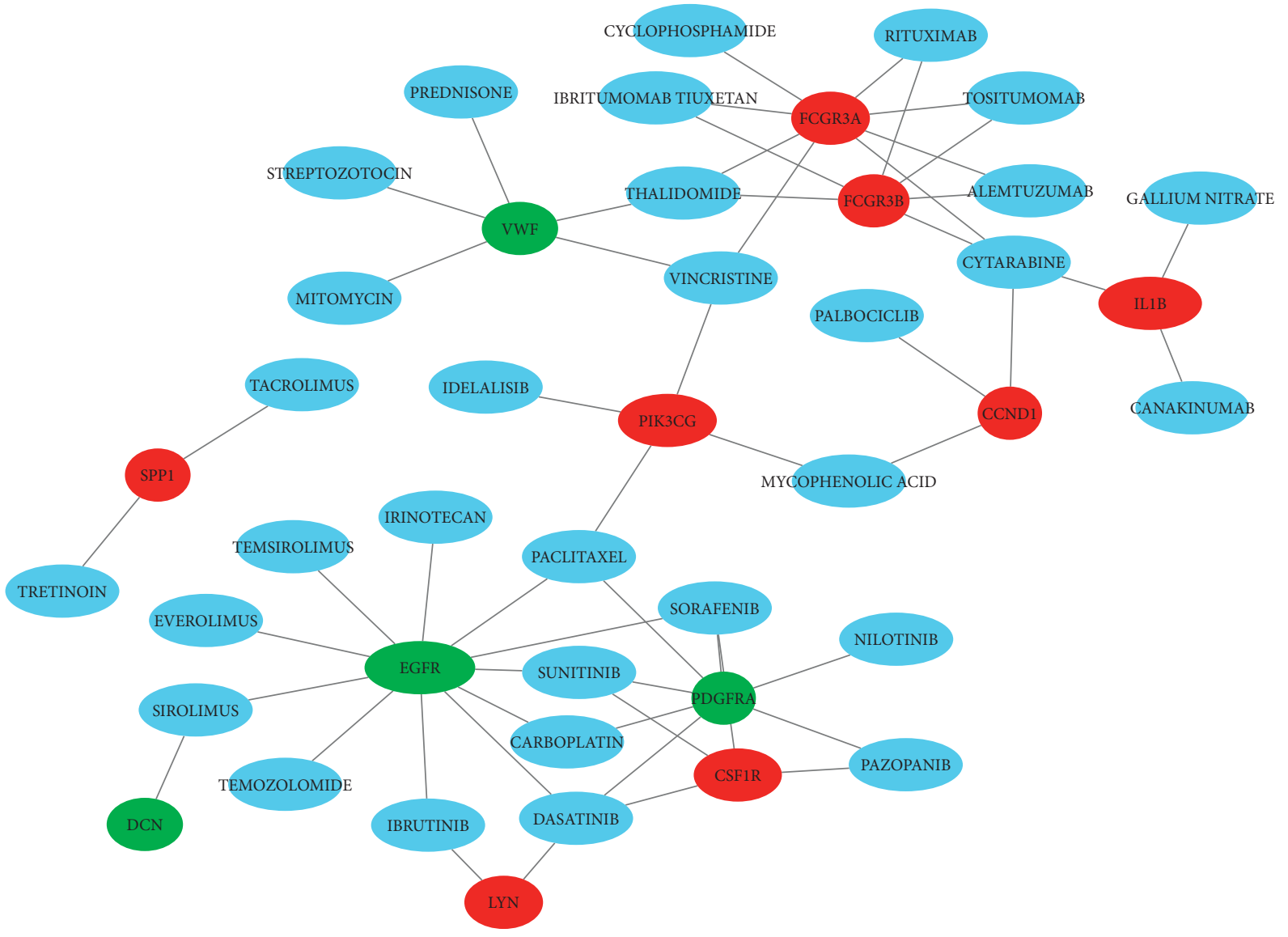

FIgURE 3: Drug-gene interactions of hub genes.

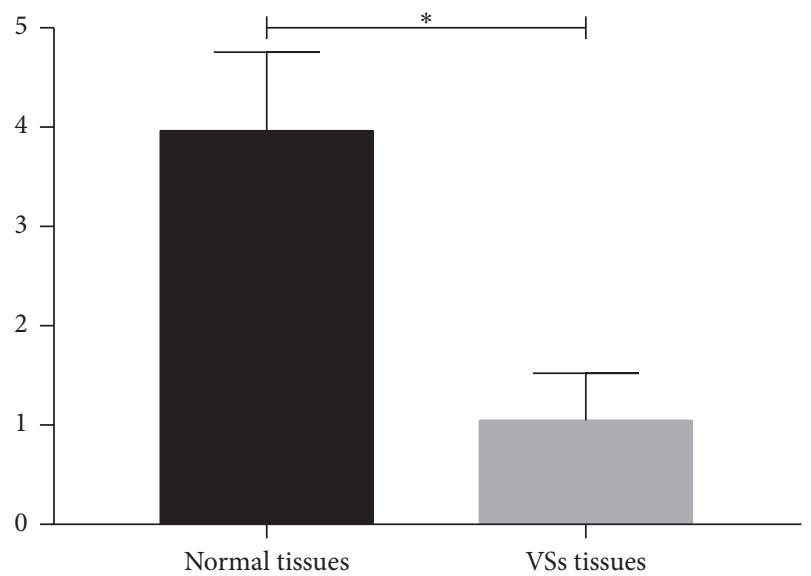

FIgURe 4: The mRNA expression levels of EGFR $\left({ }^{*} P<0.05\right)$.

prognosis in NF2-associated VSs. The drugs that inhibit the PI3K-Akt signaling pathway may be a potential therapeutic strategy for NF2 by antitumor activity against NF2-related tumor cells.

Merlin, a tumor suppressor, is constantly inactivated in NF2-associated VSs. SPP1, also known as osteopontin (OPN), is a secreted, integrin-binding phosphoprotein. OPN had been insufficiently studied in VSs, while elevated OPN is a utility of some tumors progression and metastasis, suggesting a poor prognosis, such as breast cancer [7]. Morrow et al. study [7] revealed that OPN-initiated signaling induced Akt-mediated phosphorylation and degradation of merlin in breast cancer cells; it was reported for the first time that OPN is involved in merlin protein degradation. We showed that SPP1 is upregulated in NF2-associated VSs, consistent with the result of Torres-Martin et al. [8]. SPP1 may be a biomarker of NF2-associated VSs, whose interaction with merlin has not been reported in NF2-associated VSs. Furthermore, we found that drugs associated with SPP1, including tacrolimus and tretinoin, may be potential therapeutic agents for NF2-associated VSs, which require a one-step study. Tacrolimus, a powerful immunosuppressant, significantly increased OPN mRNA and protein expression from kidney tissue and renal cells, which may contribute to nephrotoxicity inducing [9]. However, tacrolimus used to treat autoimmunity blocks IL2 production and is used for active rheumatoid arthritis [10] and lupus nephritis [11]. Based on functional annotation and pathway enrichment analysis of DEGs, inflammatory response, immune response, melanoma, and rheumatoid arthritis may be connected with NF2-associated VSs development. Therefore, tacrolimus may be used for NF2-associated VSs treatment.

In our study, CCND1 involved in apoptosis and cell cycle control, a key cell cycle regulatory protein, was upregulated 
in NF2-associated VSs, which is consistent with previous studies $[12,13]$. Elevated CCND1 is known to suggest poor prognosis in many cancers, such as colorectal cancer [14], breast cancer [15], and multiple myeloma [16, 17]. We found drugs associated with CCND1, including palbociclib and mycophenolic acid, which had not been studied in VSs. Palbociclib, a cyclin-dependent kinase 4 and 6 (CDK4/6) inhibitor, prolongs progression-free survival among patients with advanced estrogen receptor-positive and HER2-negative breast cancer $[18,19]$. Mycophenolic acid, an immunosuppressant, can inhibit proliferation and induce apoptosis in cancer cells, which may be caused by inhibition of upregulation of CCND1 and the PI3K/AKT/mTOR pathway [20]. Very interestingly, CCND1 was also upregulated in NF2-associated VSs and was significantly enriched in the PI3K-Akt signaling pathway in this study. Thus, palbociclib and mycophenolic acid may inhibit the growth of NF2-associated VSs.

In contrast to SPP1 and CCND1, EGFR was downregulated in NF2-associated VSs, in agreement with the results of Torres-Martin et al. [8], but contrary to those of Yi et al. [21]. At present, the efficacy of EGFR inhibitors in acoustic neuroma treatment is not ideal yet, which may be related to EGFR downregulated in some patients.

In conclusion, with the present analysis, we identified 28 drugs not yet tested in NF2-associated VSs. Tacrolimus, palbociclib, and mycophenolic acid may be candidate drugs. SPP1 and CCND1 may be potential targeted genes in NF2associated VSs. PI3K-Akt signaling pathway may be involved in VSs development.

\section{Data Availability}

The data used to support the findings of this study are available from the corresponding author upon request.

\section{Conflicts of Interest}

All authors declare that they have no conflicts of interest.

\section{Acknowledgments}

The authors greatly appreciate the financial support from the General Program of Natural Science Foundation of Guangxi Province of China (no. 2016GXNSFAA380150) and NSFC Cultivation Project of the Second Affiliated Hospital of Guangxi Medical University (GJPY2019001).

\section{References}

[1] J. O. Blakeley, X. Ye, D. G. Duda et al., "Efficacy and biomarker study of bevacizumab for hearing loss resulting from neurofibromatosis type 2-associated vestibular schwannomas," Journal of Clinical Oncology, vol. 34, no. 14, pp. 1669-1675, 2016.

[2] A. Meola and S. D. Chang, "Bilateral vestibular schwannomas in neurofibromatosis type 2," New England Journal of Medicine, vol. 379, no. 15, p. 1463, 2018.

[3] F. Zhao, B. Wang, Z. Yang et al., "Surgical treatment of large vestibular schwannomas in patients with neurofibromatosis type 2: outcomes on facial nerve function and hearing preservation," Journal of Neuro-Oncology, vol. 138, no. 2, pp. 417-424, 2018.

[4] K. Tanaka, A. Eskin, F. Chareyre et al., "Therapeutic potential of HSP90 inhibition for neurofibromatosis type 2," Clinical Cancer Research, vol. 19, no. 14, pp. 3856-3870, 2013.

[5] S. Agnihotri, I. Gugel, M. Remke et al., "Gene-expression profiling elucidates molecular signaling networks that can be therapeutically targeted in vestibular schwannoma," Journal of Neurosurgery, vol. 121, no. 6, pp. 1434-1445, 2014.

[6] L. Provenzano, Y. Ryan, D. A. Hilton et al., "Cellular prion protein (PrPC) in the development of merlin-deficient tumours," Oncogene, vol. 36, no. 44, pp. 6132-6142, 2017.

[7] K. A. Morrow, S. Das, B. J. Metge et al., "Loss of tumor suppressor merlin in advanced breast cancer is due to posttranslational regulation," Journal of Biological Chemistry, vol. 286, no. 46, pp. 40376-40385, 2011.

[8] M. Torres-Martin, L. Lassaletta, J. San-Roman-Montero et al., "Microarray analysis of gene expression in vestibular schwannomas reveals SPP1/MET signaling pathway and androgen receptor deregulation," International Journal of Oncology, vol. 42, no. 3, pp. 848-862, 2013.

[9] A. Khanna, "Tacrolimus and cyclosporinein vitro and in vivo induce osteopontin mRNA and protein expression in renal tissues," Nephron Experimental Nephrology, vol. 101, no. 4, pp. e119-e126, 2005.

[10] M. P. Curran and C. M. Perry, “Tacrolimus,” Drugs, vol. 65, no. 7, pp. 993-1001, 2005, discussion 1002-1003.

[11] C. C. Mok, K. Y. Ying, C. W. Yim et al., "Tacrolimus versus mycophenolate mofetil for induction therapy of lupus nephritis: a randomised controlled trial and long-term followup," Annals of the Rheumatic Diseases, vol. 75, no. 1, pp. 30-36, 2016.

[12] P. Rajaraman, S. S. Wang, N. Rothman et al., "Polymorphisms in apoptosis and cell cycle control genes and risk of brain tumors in adults," Cancer Epidemiology Biomarkers \&amp; Prevention, vol. 16, no. 8, pp. 1655-1661, 2007.

[13] J. Jabbour, P. Earls, N. Biggs, G. Gracie, P. Fagan, and R. Bova, "role of cyclins D1 and D3 in vestibular schwannoma," The Journal of Laryngology \& Otology, vol. 130, no. S1, pp. S2-S10, 2016.

[14] M. Xie, F. Zhao, X. Zou, S. Jin, and S. Xiong, "The association between CCND1 G870A polymorphism and colorectal cancer risk: a meta-analysis," Medicine (Baltimore), vol. 96, no. 42, p. e8269, 2017.

[15] M. A. Bewick, M. S. C. Conlon, and R. M. Lafrenie, "Polymorphisms in XRCC1, XRCC3, and CCND1 and survival after treatment for metastatic breast cancer," Journal of Clinical Oncology, vol. 24, no. 36, pp. 5645-5651, 2006.

[16] S. Manier, D. Huynh, Y. J. Shen et al., "Inhibiting the oncogenic translation program is an effective therapeutic strategy in multiple myeloma," Science Translational Medicine, vol. 9, no. 389, 2017.

[17] V. Shah, A. L. Sherborne, B A. Walker et al., "Prediction of outcome in newly diagnosed myeloma: a meta-analysis of the molecular profiles of 1905 trial patients," Leukemia, vol. 32, no. 1, pp. 102-110, 2018.

[18] N. C. Turner, D. J. Slamon, J. Ro et al., "Overall survival with palbociclib and fulvestrant in advanced breast cancer," New England Journal of Medicine, vol. 379, no. 20, pp. 1926-1936, 2018.

[19] L. Malorni, G. Curigliano, A. M. Minisini et al., "Palbociclib as single agent or in combination with the endocrine therapy received before disease progression for estrogen receptor- 
positive, HER2-negative metastatic breast cancer: TREnd trial," Annals of Oncology, vol. 29, no. 8, pp. 1748-1754, 2018.

[20] S. Domhan, C. Schwager, Q. Wei et al., "Deciphering the systems biology of mTOR inhibition by integrative transcriptome analysis," Current Pharmaceutical Design, vol. 20, no. 1, pp. 88-100, 2014.

[21] D. Yi, S. Z. Kuo, H. Zheng et al., "Activation of PDGFR and EGFR promotes the acquisition of a stem cell-like phenotype in schwannomas," Otology \& Neurotology, vol. 33, no. 9, pp. 1640-1647, 2012. 